\title{
UNIVERSAL INEQUALITIES FOR EIGENVALUES OF THE LAMÉ SYSTEM
}

\author{
He-Jun Sun And Ye-JuAn PEnG
}

\begin{abstract}
In this paper, we investigate the Dirichlet eigenvalue problem of the Lamé system: $\Delta \mathbf{u}+\alpha \operatorname{grad}(\operatorname{div} \mathbf{u})=-\sigma \mathbf{u}$ on a bounded domain $\Omega$ in an $n$-dimensional Euclidean space $\mathbb{R}^{n}$, where $\alpha$ is a nonnegative constant and $\mathbf{u}$ is a vector-valued function on $\Omega$. We establish a Levitin-Parnovski-type inequality for its eigenvalues, which gives an estimate for the upper bounds of $\sum_{i=1}^{n} \sigma_{i+j}$ for any positive integer $j$. Moreover, we obtain some other universal inequalities for eigenvalues of this problem.
\end{abstract}

Mathematics subject classification (2010): 35P15, 35J47.

Keywords and phrases: Lamé system, eigenvalue, universal inequality.

\section{REFERENCES}

[1] M. S. Ashbaugh And R. D. Benguria, A second proof of the Payne-Pólya-Weinberger conjecture, Commun. Math. Phys. 147 (1992), 181-190.

[2] M. S. Ashbaugh And R. D. Benguria, More bounds on eigenvalue ratios for Dirichlet Laplacians in $n$ dimension, SIAM J. Math. Anal. 24 (1993), 1622-1651.

[3] D. G. Chen And Q.-M. Cheng, Extrinsic estimates for eigenvalues of the Laplace operator, J. Math. Soc. Japan 60 (2008), 325-339.

[4] D. G. Chen, Q.-M. Cheng, Q. L. WANG, And C. Y. XIA, On eigenvalues of a system of elliptic equations and of the biharmonic operator, J. Math. Anal. Appl. 387 (2012), 1146-1159.

[5] Q.-M. CHENG AND H. C. YANG, Universal inequalities for eigenvalues of a system of elliptic equations, P. Roy. Soc. Edinb. 139A (2009), 273-285.

[6] S. M. HooK, Domain independent upper bounds for eigenvalues of elliptic operators, Trans. Amer. Math. Soc. 318 (1990), 615-642.

[7] S. M. HooK, Bounds for the fundamental frequencies of an elastic medium, Michigan Math. J. 38 (1991), 3-12.

[8] S. Ilias AND O. MAKHOUL, Universal inequalities for the eigenvalues of a power of the Laplacian operator, Manuscripta Math. 132 (2010), 75-102.

[9] B. KAWOHL AND G. SWEEGS, Remarks on eigenvalues and eigenfunctions of a special elliptic system, J. Appl. Math. Phys. (ZAMP) 38 (1987), 730-740.

[10] H. A. LEVINE AND M. H. PROTTER, Unrestricted lower bounds for eigenvalues of elliptic equations and systems of equations with applications to problems in elasticity, Math. Meth. Appl. Sci. 7 (1985), 210-222.

[11] M. LeVITIN AND L. PARnovs KI, Commutators, spectral trace identities, and universal estimates for eigenvalues, J. Funct. Anal. 192 (2002), 425-445.

[12] S. Mayboroda And M. Mitrea, The poisson problem for the Lamé system on low-dimensional Lipschitz domains, in: Integral Methods in Science and Engineering, C. Constanda, Z. Nashed and D. Rollins eds., Birkhaüser, 2006, pp. 137-160.

[13] L. E. Payne, G. Pólya, And H. F. Weinberger, On the ratio of consecutive eigenvalues, J. Math. Phys. 35 (1956), 289-298.

[14] A. Pleijel, Proprietés asymptotique des fonctions fondamentales du problems des vibrations dans un corps élastique, Arkiv f. Mat. Astr. o. Fysik 26 (1939), 1-9. 
[15] H. J. Sun, Q.-M. Cheng AND H. C. YAng, Lower order eigenvalues of Dirichlet Laplacian, Manuscripta Math. 125 (2008), 139-156.

[16] H. TRIEBEL, Höhere Analysis, VEB Deutscher Verlag der Wissenschaften, Berlin, 1972. 